\title{
Multimodal Image Driven Patient Specific Tumor Growth Modeling
}

\author{
Yixun Liu ${ }^{1}$, Samira M. Sadowski ${ }^{2}$, Allison B. Weisbrod ${ }^{2}$, Electron Kebebew ${ }^{2}$, \\ Ronald M. Summers ${ }^{1}$, and Jianhua $\mathrm{Yao}^{1}$ \\ ${ }^{1}$ Radiology and Imaging Sciences, NIH \\ ${ }^{2}$ Endocrine Oncology Branch, National Cancer Institute, NIH
}

Personalized tumor growth model using clinical imaging data is valuable in tumor staging and therapy planning. In this paper, we build a patient specific tumor growth model based on longitudinal dual phase CT and FDG-PET. We propose a reactionadvection-diffusion model integrating cancerous cell proliferation, infiltration, metabolic rate and extracellular matrix biomechanical response. We then develop a scheme to bridge our model with multimodal radiologic images through intracellular volume fraction (ICVF) and Standardized Uptake Value (SUV). The model was evaluated by comparing the predicted tumors with the observed tumors in terms of average surface distance (ASD), root mean square difference (RMSD) of the ICVF map, the average ICVF difference (AICVFD) of tumor surface and the tumor relative volume difference (RVD) on six patients with pathologically confirmed pancreatic neuroendocrine tumors. The ASD between the predicted tumor and the reference tumor was $2.5 \pm 0.7 \mathrm{~mm}$, the RMSD was $4.3 \pm 0.6 \%$, the AICVFD was $2.6 \pm 0.8 \%$, and the RVD was $7.7 \pm 1.9 \%$.

\section{Introduction}

Quantitatively characterizing the tumor spatial-temporal progression is valuable in staging tumor and designing optimal treatment strategies. Tumor growth not only relies on the properties of cancer cells, but also depends on dynamic interactions among cancer cells, and between cells and their constantly changing microenvironment. The complexity of the cancer system motivates the study of the tumor growth using mathematical models [1] [2] [3]. Swanson et al. [1] assumed an infiltrative growth of the tumor cells, while considering differences in cell diffusion in white and gray matter. Clatz et al. [2] modeled locally anisotropic migration patterns by integrating information from diffusion tensor images (DTI). Hogea et al. [3] included the mechanical properties of the lesion on surrounding structures to model mass effect.

All these works use a reaction-diffusion model, originally introduced by Turing over 60 years ago, to study cell proliferation and infiltration. In this work, we will extend the reaction-diffusion model to incorporate cell metabolic rate based on the energy conservation law. The travelling front of the reaction-diffusion model offers the benefit that the model prediction (front) can be directly connected with the anatomical CT and MRI via the identifiable tumor boundary in the image. However, tumor boundary only provides limited tumor physiological information. In this paper, 
we extract underlying tumor cell fraction in each image voxel using dual phase CT image and incorporate it into the growth model.

In this paper, we target image driven patient specific tumor modeling using routine clinical CT and FDG-PET data. To bridge the gap between the model and the imaging data, we introduce energy conservation law into the modeling and developed a reaction-advection-diffusion model to incorporate cell proliferation, infiltration, metabolic rate and mass effect. We further incorporate intracellular volume fraction (ICVF) derived from dual phase CT data and glucose metabolic rate from FDG-PET (2-[18F] Fluoro-2-deoxyglucose positron emission tomography) to measure model physiological parameters.

We evaluate the proposed model on pancreatic neuroendocrine tumor. The only work on the pancreatic tumor modeling that we are aware of is [4], in which the authors used a compartment model to divide the cell population into three subpopulations: primary tumor cells, metastasis-enabled cells and metastasized cells. The migration rate between subpopulations and the growth rate and death rate within each subpopulation were estimated based on autopsy data. In this paper, we focus on the way to combine routine clinical multimodal images to study the growth of the primary solid tumor.

\section{Method}

In this section, we first derive a reaction-advection-diffusion model incorporated with cell metabolic rate via energy conservation law, and then describe how to adapt the model to associate it with routine dual phase CT and FDG-PET.

\subsection{Tumor Growth Model}

According to the tumor logistical growth law presented in [1], the number of the newly created cells can be described by,

$$
\frac{d N}{d t}=\rho N\left(1-\frac{N}{K}\right)
$$

where $N$ is the number of cells, a function of time $t . \rho$ is spatial-temporal invariant proliferation rate. This law describes that the tumor grows exponentially at the beginning and then gradually slows down as approaching the carrying capacity $K(N<K)$.

As a tumor progresses, the parts with sufficient nutrients and oxygen grow faster, and those suffering vascular inefficiencies will develop into necrosis, suggesting a heterogeneous or spatial-temporal varying proliferation function $\rho(\boldsymbol{x}, t)$. The metabolic energy conservation law presented by West et al. [5] quantitatively describes the relationship between the metabolic energy and the ontogenetic growth, providing the theoretical foundation to explore the heterogeneity of the proliferation rate. The energy conservation law states that the incoming energy $B(t)$ required for tumor growth is allocated to two parts,

$$
B=N B_{c}+E_{c} \frac{d N}{d t}
$$


where the first term represents the energy to maintain the existing cells and the second term represents the energy to create new cells. $B_{c}$ is the metabolic rate of a single cell, and $E_{c}$ is the energy required to create a cell. Both $B_{c}$ and $E_{c}$ are assumed constant during tumor growth. Replace $\frac{d N}{d t}$ in equation (2) with $\rho N\left(1-\frac{N}{K}\right)$,

$$
B=N B_{c}+E_{c} \rho N\left(1-\frac{N}{K}\right) \Rightarrow \rho=\frac{K B-K B_{c} N}{E_{c} N(K-N)}
$$

The proliferation rate $\rho$ in equation (3) is a function of time $t$. However, in clinical practice, $\rho$ is only available at specific time points when $B$ and $N$ are measurable. Thus, we approximate $\rho$ at time $t$ between 0 and $T$ through linear interpolation,

$$
\begin{gathered}
\rho(t)=\rho(0)+\frac{t}{T}(\rho(T)-\rho(0))= \\
\frac{K B_{0}-K B_{C} N_{0}}{E_{C} N_{0}\left(K-N_{0}\right)}+\frac{t}{T}\left(\frac{K B_{T}-K B_{C} N_{T}}{E_{C} N_{T}\left(K-N_{T}\right)}-\frac{K B_{0}-K B_{C} N_{0}}{E_{C} N_{0}\left(K-N_{0}\right)}\right)
\end{gathered}
$$

where $\mathrm{B}_{0}, B_{T}, N_{0}$ and $N_{T}$ are the measured metabolic rate and cell numbers at time 0 and $T$, respectively. Apply model (1) to each voxel (millions of cells within $1 \mathrm{~mm}^{3}$ ) at position $\boldsymbol{x}$, and add a diffusion term as that in the reaction-diffusion model [1] to account for cancerous cell infiltration into surrounding tissues, leading to a reactiondiffusion model,

$$
\frac{\partial N}{\partial t}=D \nabla^{2} N+\rho N\left(1-\frac{N}{K}\right)
$$

where the first term is the diffusion term, and the second term is the reaction (proliferation) term. $D$ is the diffusivity or infiltration rate. Equation (5) describes that the rate of cell number change equals the sum of the net dispersal of cancerous cells and the net proliferation of cancerous cells. Note that both $N$ and $\rho$ are a function of position $\boldsymbol{x}$ and time $t$. The cell number at position $\boldsymbol{x}$ is not only affected by the proliferation and diffusion (Brownian movement) but also affected by the underlying mechanical deformation (so-called mass effect), which is caused by the growing cells impacting on the extracellular matrix. An advection term is added into model (5) to account for the tumor cells being displaced as a consequence of the underlying mechanical deformation [3],

$\begin{array}{ll}\frac{\partial N}{\partial t}=D \nabla^{2} N-\nabla \cdot(N \boldsymbol{v})+\rho N\left(1-\frac{N}{K}\right) & \text { reaction - advection - diffusion } \\ \nabla \cdot \boldsymbol{\sigma}+\boldsymbol{b}=\mathbf{0} & \text { momentum }\end{array}$

$\boldsymbol{\sigma}=(\lambda \nabla \cdot \boldsymbol{u})+\mu\left(\nabla \boldsymbol{u}+\nabla \boldsymbol{u}^{T}\right) \quad$ constitutive

$\boldsymbol{v}=\frac{\partial \boldsymbol{u}}{\partial t} \quad$ kinematics

where the tumor cell drift velocity $\boldsymbol{v}$ depends on the displacement vector $\boldsymbol{u}$ induced by the balance between Cauchy stress tensor $\boldsymbol{\sigma}$ and body force $\boldsymbol{b}$. $\lambda$ and $\mu$ are unknown Lame's coefficients in linear elasticity. The body force $\boldsymbol{b}$ originated from the growing cells is proportional to the local gradient of the tumor cell density,

$$
\boldsymbol{b}=-p \nabla N
$$

where $p$ is an unknown positive constant. 
Model (6) allows the incorporation of cell metabolic rate and accounts for cell proliferation, infiltration, metabolism and mass effect, but not directly connected to clinical imaging data. We will further develop model (6) in the following two sections in order to associate the model parameters with measurements from CT and FDG-PET. The proposed model accounts for the cell metabolic rate and directly connects to routine CT and FDG-PET, which makes our model fundamentally novel and different from the model in [3].

\subsection{Associate the Model with Dual Phase CT}

Tissues within a voxel are considered to be made of three well known compartments: (1) a vascular space through which the blood flows; (2) an extravascular extracellular space (EES) which provides the supporting structure of the tissues; and (3) the cellular space. The extracellular volume fraction (ECVF) of the studied voxel at position $\boldsymbol{x}$ and time $t$ can be estimated by equation (8) using dual phase CT [6],

$$
\operatorname{ECVF}(\boldsymbol{x}, t)=\frac{H U_{\text {post }}(\boldsymbol{x}, t)-H U_{\text {pre }}(\boldsymbol{x}, t)}{\left(H U_{\text {post_ploodpool }}-H U_{\text {pre_ploodpool }}\right) /\left(1.0-H c t_{\text {blood }}\right)}
$$

where the numerator is the HU enhancement brought by the contrast distributed in the extracellular space (EES plus vascular space) of the studied voxel, and the denominator is the HU enhancement brought by the contrast distributed in the whole space (EES plus vascular space plus cellular space) of the reference blood pool voxel. The ratio of the HU enhancement is a measure of the fraction of the extracellular space, i.e., ECVF because the HU enhancement is proportional to the volume of the space, in which the contrast reaches equilibrium. $H U_{\text {post }}$ and $H U_{\text {pre }}$ are $\mathrm{HU}$ of the post-contrast CT image and the pre-contrast $\mathrm{CT}$ image, respectively. $H U_{\text {post_ploodpool }}$ and $H U_{\text {pre_ploodpool }}$ are average $\mathrm{HU}$ of the blood pool of the post-contrast $\mathrm{CT}$ and pre-contrast CT, respectively. The hematocrit $H c t_{\text {blood }}$ is the volume percentage (\%) of red blood cells in blood, which varies from patient to patient, but can be measured by the blood sample.

ECVF's complement ICVF can be calculated by,

$$
\theta(\boldsymbol{x}, t)=1.0-\operatorname{ECVF}(\boldsymbol{x}, t)
$$

In equation (6), the cell number $N$ is difficult to be directly measured by CT image. We adapt the reaction-advection-diffusion equation by replacing $N(\boldsymbol{x}, t)$ with $K \theta(\boldsymbol{x}, t)$ based on the assumption that all cells have similar volumes,

$$
\frac{\partial \theta}{\partial t}=D \nabla^{2} \theta-\nabla \cdot(\theta v)+\rho \theta(1-\theta)
$$

where (replace $N_{0}$ and $N_{T}$ in (4) with $K \theta_{0}$ and $K \theta_{T}$, respectively)

$$
\rho=\frac{B_{0}-a \theta_{0}}{b \theta_{0}-b \theta_{0}^{2}}+\frac{t}{T}\left(\frac{B_{T}-a \theta_{T}}{b \theta_{T}-b \theta_{T}^{2}}-\frac{B_{0}-a \theta_{0}}{b \theta_{0}-b \theta_{0}^{2}}\right)
$$

where $a=K B_{c}$ and $b=K E_{c}$. Both parameters $a$ and $b$ have specific biological meanings, representing the energy to maintain $K$ cells and create $K$ cells, respectively. Similarly, replacing $N$ in (7) with $K \theta$ leads to $\boldsymbol{b}=-p K \nabla \theta=-q \nabla \theta$. 


\subsection{Associate the Model with FDG-PET}

Normally, when the scanning time is longer than 45 min post-injection, the glucose metabolic rate $M R_{g l c}$ or $B$ can be approximated by [7],

$$
B=M R_{g l c}=\frac{\frac{G l c}{100.0} \cdot \operatorname{PET}(t) /\left(\frac{\text { dose }}{\text { bodyweight }}\right)}{L C \cdot k / 100.0} \Rightarrow B=M R_{g l c}=c \times \operatorname{SUV}(t)
$$

where $G l c$ is the glucose concentration in arterial plasma. PET $(t)$ denotes the radioactive tracer $\mathrm{FDG}^{18}$ concentration in tissue at time $t$ that is measurable from PET. $k$ is a constant that is not dependent on the particular subject being studied. $c=\frac{1.0}{L C \cdot k / 100.0}$, a lumped unknown parameter. The numerator in (12) is widely used as standardized uptake value (SUV), which is proportional to $M R_{g l c}$ since both $k$ and $L C$ are constants [7].

Replace $B_{0}$ and $B_{T}$ in equation (11) with $c \times S U V_{0}$ and $c \times S U V_{T}$, respectively,

$$
\rho=\frac{\alpha S U V_{0}-\beta \theta_{0}}{\theta_{0}-\theta_{0}^{2}}+\frac{t}{T}\left(\frac{\alpha S U V_{T}-\beta \theta_{T}}{\theta_{T}-\theta_{T}^{2}}-\frac{\alpha S U V_{0}-\beta \theta_{0}}{\theta_{0}-\theta_{0}^{2}}\right)
$$

where $\alpha=c / b, \beta=a / b$.

Equation (6) with its reaction-advection-diffusion equation replaced with equation (10) constitutes our proposed model (or state equations from an optimal control standpoint [8]). The proposed model constitutes the forward problem with unknown control parameter $\boldsymbol{g}=(\alpha, \beta, D, \lambda, \mu, q)$, which can be estimated by fitting the model predicted ICVF $\theta$ (a function of $\boldsymbol{g}$ ) with the observed ICVF $\theta_{T}$,

$$
\mathcal{J}(\boldsymbol{g})=\int_{\Omega}\left(\theta(\boldsymbol{x})-\theta_{T}(\boldsymbol{x})\right)^{2} d \boldsymbol{x}+\gamma\left(\boldsymbol{g}-\boldsymbol{g}_{\boldsymbol{r}}\right) \cdot\left(\boldsymbol{g}-\boldsymbol{g}_{r}\right)
$$

where the first term measures the degree of similarity, and the second term is Tikhonov regularization term to recover a locally unique solution close to a reference solution $\boldsymbol{g}_{r}=\left(\alpha_{r}, \beta_{r}, D_{r}, \lambda_{r}, \mu_{r}, q_{r}\right)$ defined as: $\alpha_{r}=2.3 \times 10^{-3} g^{-1} \cdot m l \cdot d a y^{-1}, \beta_{r}=1.9 \times$ $10^{-2}$ day $^{-1}, D_{r}=0.13 \mathrm{~mm}^{2} /$ day, $\lambda_{r}=9310 \mathrm{~Pa}, \mu_{r}=1034 \mathrm{~Pa}, q_{r}=200 \mathrm{~Pa}$. The reference solution was derived from literature and was just a rough estimation of the real solution. It is not necessary to be accurate since it is only used to define a region in which the real solution is located. $\gamma$ controls the balance of these two terms, which is obtained by a trial-and-error strategy. Functional (14) along with model (state) equations constitutes a coupled PDE-constrained optimization problem, which is solved by the one-shot method presented in [8].

\section{Results}

To study tumor growth, we have developed a dedicated protocol spanning for several years to collect patients with pancreatic tumors. The desirable longitudinal data needs to satisfy the requirements: 1) the tumor should be big enough (volume $>20 \mathrm{~mm}^{3}$ ) to allow us to ignore the error induced by segmentation and registration, 2) at least three time points and each time point includes both dual-phase CT and FDG-PET, and 3) without any treatments. Usually, a tumor will be surgically removed when it becomes sufficiently big. The contradictive requirements 1) and 3) lead to the difficulty to obtain desirable data. 
We evaluate the proposed model by comparing the predicted ICFV and tumor with the measured ICVF and tumor at the $2^{\text {nd }}$ follow-up. The predicted ICVF was produced by growing the ICVF (using model equation) from the $1^{\text {st }}$ follow-up for the period between the $1^{\text {st }}$ and $2^{\text {nd }}$ follow-up with the parameters estimated from the longitudinal data at the baseline and the $1^{\text {st }}$ follow-up. The predict tumor is an isosurface extracted from the predicted ICVF based on a threshold. Six patients with pathologically confirmed untreated pancreatic neuroendocrine tumors were enrolled in our experiment. Fig.1 shows the longitudinal post-contrast CT, fused PET/CT, estimated SUV map (with decay correction) and the ICVF maps (only the relative heterogeneous ICVF region is shown).

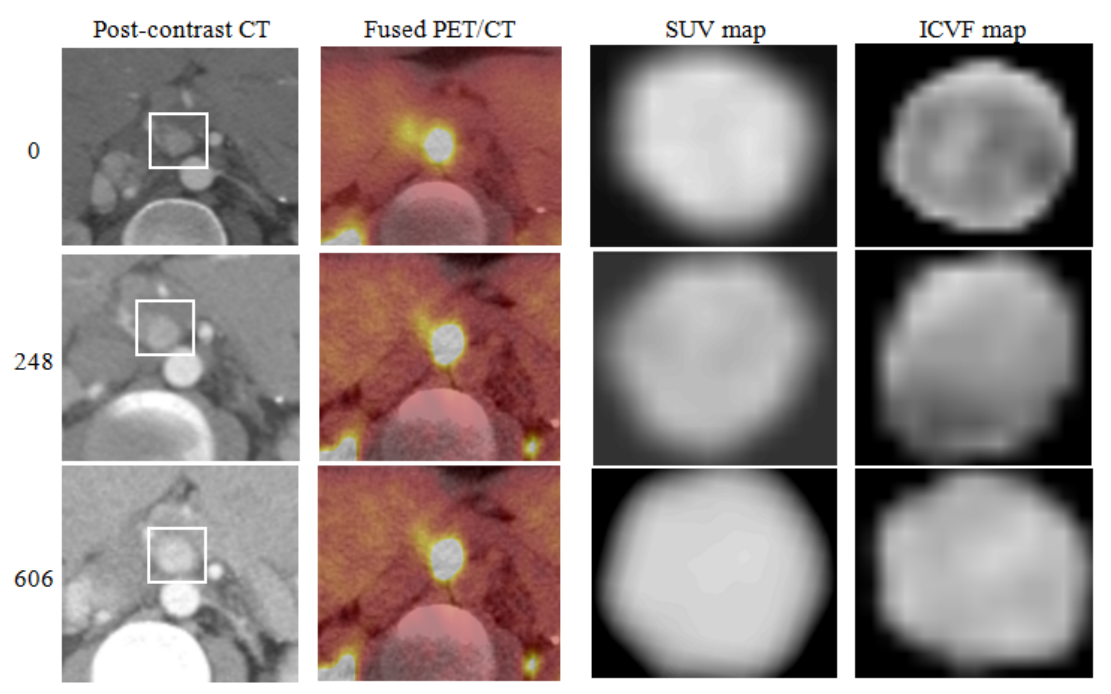

Fig. 1. Longitudinal original and intermediate results. The rows correspond with baseline $(T=0$ day), $1^{\text {st }}$ follow-up ( $T=248$ days), and $2^{\text {nd }}$ follow-up ( $T=606$ days) and the columns correspond with post-contrast CT, fused PET/CT, estimated SUV and ICVF maps. The white bounding box highlights the tumor.

Fig. 2 shows the comparison between the reference results of the $2^{\text {nd }}$ follow-up and the prediction results of two patients. The first row demonstrates a similar distribution of the ICVF map between the reference and the prediction results for both two patients: cell number decreases from the center to the rim of the tumor. The predicted center region (blue) is more homogeneous than the reference part, which might be caused by the exclusion of the complex heterogeneous tumor microenvironment in our model. The comparison of the isocontours of the ICVF map is shown in the second row. The inner most contour shows larger discrepancy, but the outer contours agree well with each other, suggesting a more heterogeneous cell distribution in the center region of the reference tumor, which also can be observed in the gray scale ICVF (the last column of Fig.1). In the third row, we compare the ICVF on the surface of the segmented tumor in post-contrast CT of the $2^{\text {nd }}$ follow-up. We focus on this surface because it is one that can be identified in the image data with our naked eyes. Both patients show similar ICVF distribution and the second patient demonstrated a 
more homogeneous ICVF distribution on the tumor surface than the first patient. We assume the average ICVF on the tumor surface to be the threshold that defines the detectable tumor boundary, which makes sense since ICVF is a main factor affecting HU of CT. We use this threshold to extract the isosurface (predicted tumor) from the predicted ICVF map to compare with the segmented tumor in terms of average surface distance and relative volume difference.

The quantitative evaluation regarding the root mean square difference (RMSD) of ICVF map, the average ICVF difference (AICVFD) of tumor surface, the average surface distance (ASD) between the predicted tumor surface and the segmented (reference) tumor surface, and the relative tumor volume difference (RVD, ratio of the volume difference and the volume of the reference volume) are listed in Table 1 , in which the RMSD is $4.3 \pm 0.6 \%$, the AICVFD is $2.6 \pm 0.8 \%$, the ASD is $2.5 \pm 0.7 \mathrm{~mm}$, and the RVD is $7.7 \pm 1.9 \%$. The predicted ICVF value is slightly large, but the boundary prediction (clinically relevant) is very promising with an average error around $2.5 \mathrm{~mm}$. In fact, there is no work to compare with our work regarding ICVF because we are the first to introduce ICVF into the modeling.

We conducted the global nonlinear non-monotonic sensitivity analysis using extended Fourier Amplitude Sensitivity Test (eFAST). Both the first order $\left(S_{i}\right)$ and total order $\left(S_{T i}\right)$ sensitivity indexes show the biological parameters: $D, \alpha$ and $\beta$ are consistently higher than the three biomechanical parameters: $\mu, \lambda$ and $q$, which suggests the biological parameters affect the ICVF more than the biomechanical parameters. In the biological parameters, the diffusion $D$ is highest $\left(S_{i}=0.4206, S_{T i}=0.7422\right)$, probably disclosing the aggressive infiltration of the pancreatic tumor. In the biomechanical parameters, $\mu$ and $\lambda$ dominate the explanation of the variation, which makes sense since these two parameters control the stiffness and incompressibility of the tissue.

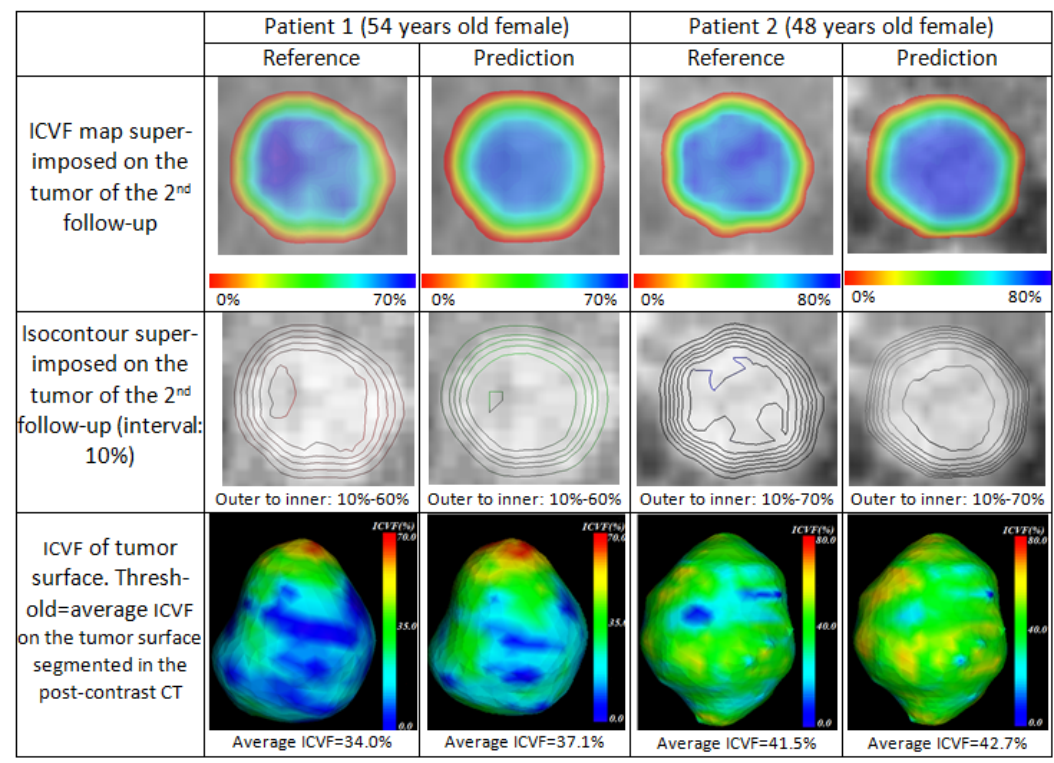

Fig. 2. Comparison between the reference (the $2^{\text {nd }}$ follow-up) and the prediction of two patients regarding ICVF map, isocontour and ICVF of tumor surface 
Table 1. Quantitative evaluation. ICVF ([min $\%$, max $\%]$ ), RMSD (\%), AICVFD (\%), ASD (mm), RVD $(\%)$. The value in the parenthesis of the ASD is the threshold to extract the predicted tumor from predicted ICVF map. $\gamma=0.6$.

\begin{tabular}{|c|c|c|c|c|c|c|}
\hline Id & ICVF & Predicted ICVF & RMSD & AICVFD & ASD & RVD \\
\hline 1 & {$[0,73]$} & {$[0,81]$} & 4.3 & 2.9 & $2.1(42.8)$ & 8.3 \\
\hline 2 & {$[0,75]$} & {$[0,88]$} & 5.2 & 2.4 & $2.5(38.1)$ & 7.6 \\
\hline 3 & {$[0,69]$} & {$[0,65]$} & 4.6 & 3.1 & $3.3(34.0)$ & 10.4 \\
\hline 4 & {$[0,75]$} & {$[0,79]$} & 3.7 & 1.2 & $3.1(41.5)$ & 8.2 \\
\hline 5 & {$[0,79]$} & {$[0,77]$} & 3.8 & 3.5 & $1.5(39.2)$ & 4.5 \\
\hline 6 & {$[0,66]$} & {$[0,78]$} & 4.1 & 2.2 & $2.4(32.2)$ & 7.1 \\
\hline mean \pm std & {$[0,73 \pm 5]$} & {$[0,81 \pm 4]$} & $4.3 \pm 0.6$ & $2.6 \pm 0.8$ & $2.5 \pm 0.7$ & $7.7 \pm 1.9$ \\
\hline
\end{tabular}

\section{Conclusions and Future Work}

In this paper, we presented a tumor growth model, which is characterized by incorporating cell metabolic rate into the reaction-diffusion model and being driven by routine clinical imaging data based on ICVF and SUV. The experiment on pancreatic neuroendocrine tumors demonstrated the promise of the proposed model. Other than the characteristics of tumor itself such as the aggressiveness measured by the metabolic rate, tumor microenvironment is also essential for the tumor growth. In the future, besides dual phase CT and FDG-PET, we will introduce DCE-MRI to measure vasculature/perfusion region and FMISO-PET to measure hypoxia region in order to capture some parts of the complex tumor microenvironment.

\section{References}

1. Swanson, K.R., Alvord, E.C., Murray, J.D.: A quantitative model for differential motility of gliomas in grey and white matter. Cell Prolif. 33, 317-329 (2000)

2. Clatz, O., Maxime, S., Pierre-Yves, B., Herve, D., Simon, K.W., Greoire, M., Nicholas, A.: Realistic simulation of the 3-D growth of brain tumors in MR images coupling diffusion with biomechanical deformation. IEEE Transactions on Medical Imaging 24, 1334-1346 (2005)

3. Hogea, C., Davatzikos, C., Biros, G.: An image-driven parameter estimation problem for a reaction-diffusion glioma growth model with mass effects. J. Math. Biol. 56, 793-825 (2008)

4. Haeno, H., Mithat, G., Meghan, B.D., Joseph, M.H., Christine, A.L., Franziska, M.: Computational modeling of Pancreatic cancer reveals kinetics of metastasis suggesting optimum treatment strategies. Cell 148, 362-375 (2012)

5. West, G.B., Brown, G.H., Enquist, B.J.: A general model for ontogenetic growth. Nature 413, 628-631 (2001)

6. Nacif, M.S., Kawel, N., Lee, J.J., Chen, X., Yao, J., Zavodni, A., Sibley, C.T., Lima, J.A., Liu, S., Bluemke, D.A.: Interstitial myocardial fibrosis assessed as extracellular volume fraction by low radiation dose cardiac CT. Radiology 264(3), 876-883 (2012)

7. Huang, S.: Anatomy of SUV. Nuclear Medicine \& Biology 27, 643-646 (2000)

8. Gunzburger, M.D.: Perspectives in Flow Control and Optimization. Advances in Design and Control, pp. 14-21. SIAM (2003) 